\title{
Post Traumatic Fat Embolism Syndrome: A Case Report
}

\author{
Moktan, Sushila Lama*, Shrestha Babu Raja \\ Kathmandu Medical College Teaching Hospital, Kathmandu, Nepal
}

*Corresponding Author: Moktan, Sushila Lama, Kathmandu Medical College Teaching Hospital, Kathmandu, Nepal.Email: sushidagreat@hotmail.com

\begin{abstract}
Fat embolism syndrome (FES) is a rare clinical syndrome characterized by signs and symptoms resulting from fat emboli mostly after orthopedic trauma. It usually occurs between 12 to 72 hours after the insult. It has high mortality and morbidity. Diagnosis of this fatal syndrome is mainly based on clinical presentation. At present, management is predominantly supportive care. Here, we report a case of FES in patient with multiple fractures of left upper and lower limb. Given the absence of a gold standard diagnostic test or pathognomonic feature, he fulfilled the criteria by Lindeque, Schonfield and Gurd. The clinical course of the disease as well as the management in the intensive care unit are discussed.
\end{abstract}

Keywords: arterial blood gas, fat embolism syndrome, intensive care unit, multiple fractures.

\section{INTRODUCTION}

Fat embolism is a process by which fat particles pass into the bloodstream and settle within a blood vessel eventually blocking a blood vessel. Fat emboli tend to be small and multiple, causing numerous signs and symptoms. Fat embolism syndrome (FES) is a serious consequence of fat emboli producing a distinct pattern of clinical symptoms and signs. FES accounts for only 2-5\% of patients who have long-bone fractures. [1]

Rates of FES in orthopedic trauma patients vary from less than 1 percent to more than 30 percent, with the wide range likely reflecting study population heterogeneity and a lack of standardization for diagnostic criteria $[2,3]$. The incidence of the fat embolism syndrome depends on the bone involved, whether fractures are isolated or multiple, the age and gender of the patient

\section{CASE REPort}

A 24 year old male was referred to our hospital with the history of head on collision between two motorbikes 2 days prior to admission. He had sustained multiple fractures (figure 1); a compound GA III A fracture of left proximal radius and ulna, fracture left clavicle and comminuted fracture of left tibia fibula. Fracture of upper and lower limb was immobilized with back slab at the previous healthcare facility.

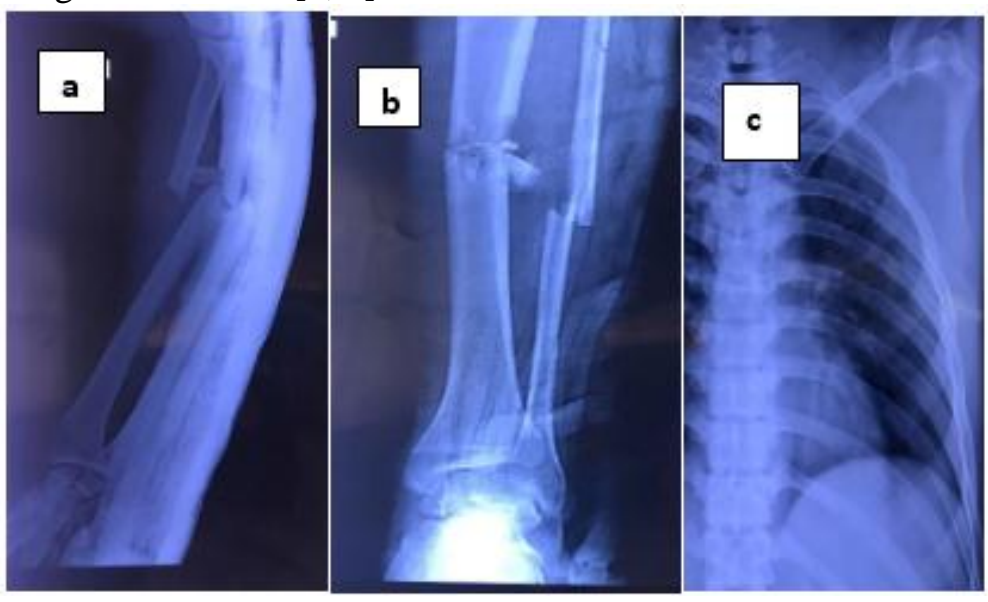

Figure1. fracture left a) radius and ulna $b$ ) tibia and fibula c) clavicle

During presentation at the emergency department, he was conscious and oriented
(Glasgow coma scale 15/15), tachycardic (heart rate 132 per min), blood pressure $156 / 98 \mathrm{mmHg}$, 
tachypnoeic (respiratory rate 36 per min) with oxygen saturation $75 \%$ in room air and afebrile. On auscultation of chest bilateral basal crepitations were heard. With Oxygen supplementation (up to $10 \mathrm{~L} \cdot \mathrm{min}-1$ ) and intravenous fluids and analgesics, patient was immediately shifted to intensive care unit after sending normal routine investigations. He was fit before the accident with no known medical illness. Chest X-ray radiography showed the appearance of ARDS (figure 2), he was planned for CT pulmonary angiogram, CT head, CECT abdomen and pelvis and $\mathrm{D}$-dimer.

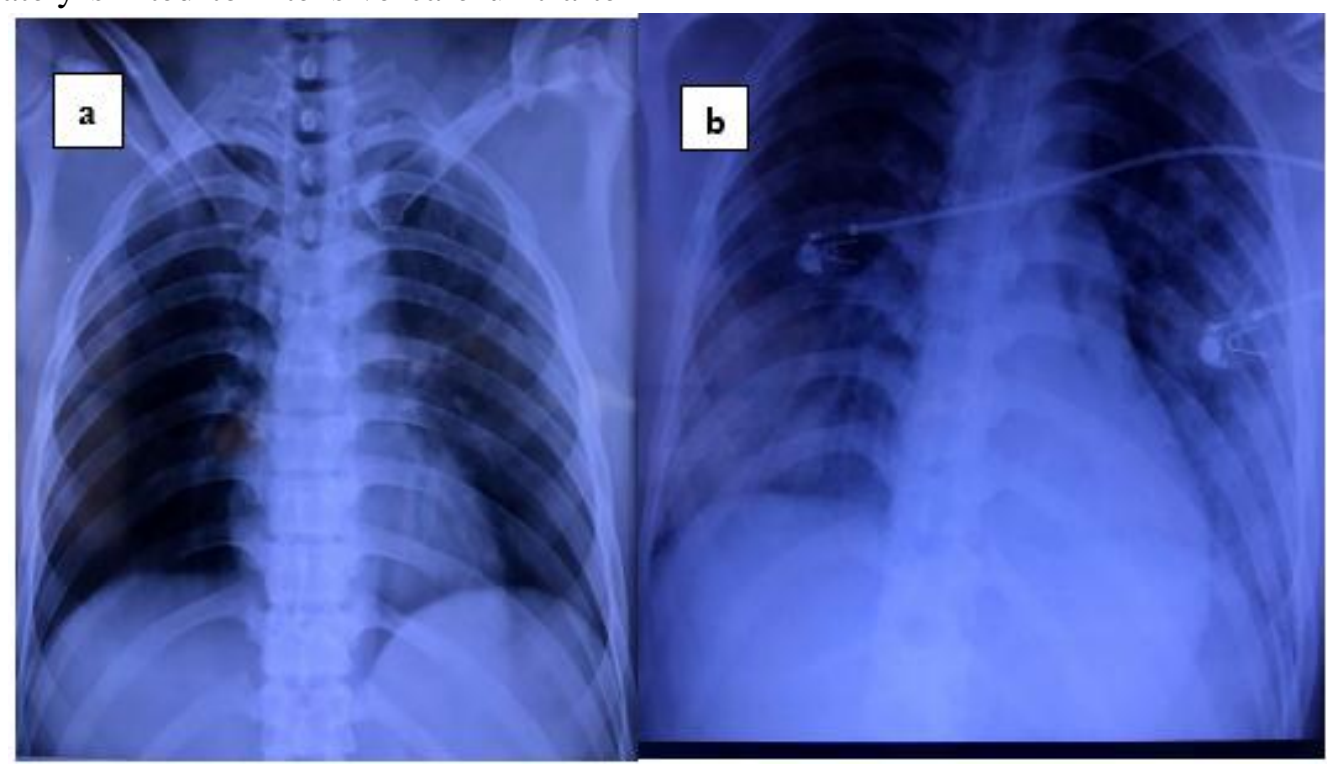

Figure2. Chest $X$-ray a) on day 1 of RTA $b$ ) AP view day 3 on admission

Patient was kept in external CPAP immediately with $100 \%$ FIO2 as his oxygen saturation was only $80 \%$ with $10 \mathrm{~L}$ of oxygen in reservoir bag, he then maintained his oxygenation with $\mathrm{PaO} 2$ of $92 \mathrm{~mm}$ of $\mathrm{Hg}$. A standard monitoring with ECG, NIBP, pulse oximeter was done and a central venous line and the arterial line was kept. Along with the fluids, antibiotics and analgesics, a low molecular weight heparin $60 \mathrm{mg}$ sub cutaneous $\mathrm{BD}$, injection methylprednisolone $1 \mathrm{mg} / \mathrm{kg} /$ day and tab.

Rosuvastatin 20mg via nasogastric tube was added. Following morning, patient was, anxious, confused, tachycardic (146/min), febrile (101 degrees Fahrenheit) and respiratory rate was up to 50 breaths per minute despite the use of Dexmedetomidine i.v infusion for sedation. His status deteriorated with oxygen saturation of $80 \%$ and $\mathrm{PaO} 2$ of $54 \mathrm{~mm}$ of $\mathrm{Hg}$ despite on $100 \% \mathrm{FiO} 2$. On auscultation of the lungs, there was bilateral decreased air entry with increasing crepitations and petechial rash was noted widespread on the anterior the chest, axilla till the umbilicus. Immediately tracheal intubated was performed and mechanical ventilation was commenced. $\mathrm{He}$ was kept on volume controlled ventilation with
Vecuronium, Dexmedetedomidine and, Midazolam infusion. But the patient still remained hypoxic revealing blood gas of ; $\mathrm{pH}$ 7.26, $\mathrm{PaCO} 2$ of $58 \mathrm{~mm}$ of $\mathrm{Hg}, \mathrm{PaO} 2$ of $60.9 \mathrm{~mm}$ of $\mathrm{Hg}$ on $100 \%$ Fio2, PEEP of 12. Only after about 6 hours the oxygen saturation raised up $92 \%$ with $\mathrm{pH}$ of $7.435, \mathrm{PaO} 274.3$ and $\mathrm{PaCo} 2$ of $48 \mathrm{~mm}$ of $\mathrm{Hg}$.

\section{FAт EMbOLISM 2}

CT pulmonary angiogram (figure 3 ) report stated low fat attenuating filling defect within the lumen of the anterior branch of descending right interlobar pulmonary artery suggestive of fat embolism. Other findings were diffuse patchy ground glass attenuation in the upper lobe of bilateral lungs and right middle lobe as well as patchy airspace opacifications in the lower lobe of bilateral lungs with displaced fracture of the left clavicle and anterior compression fracture of D6 vertebra. CT scan of the abdomen and head (figure 4) was normal, funduscopic examination of eye did not revealed any changes related to fat embolism. His D-dimer was $1.1 \mathrm{mg} / \mathrm{l}$ (reference range $<0.5$ ), serum lipase $22 \mathrm{u} / \mathrm{l}$ (ref. range $<60$ ) and fat globules were not seen in the urine. Bedside echo revealed ejection fraction of about 50 to $60 \%$ with no dilatation of the chambers. 

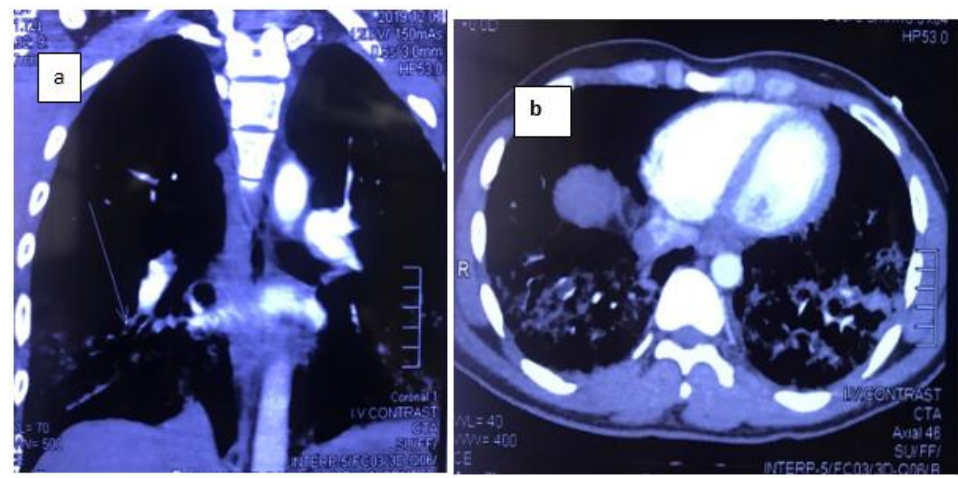

Figure3. Chest CT angiogram: a) filling defect in the artery (arrow) b) diffuse patchy ground glass appearance

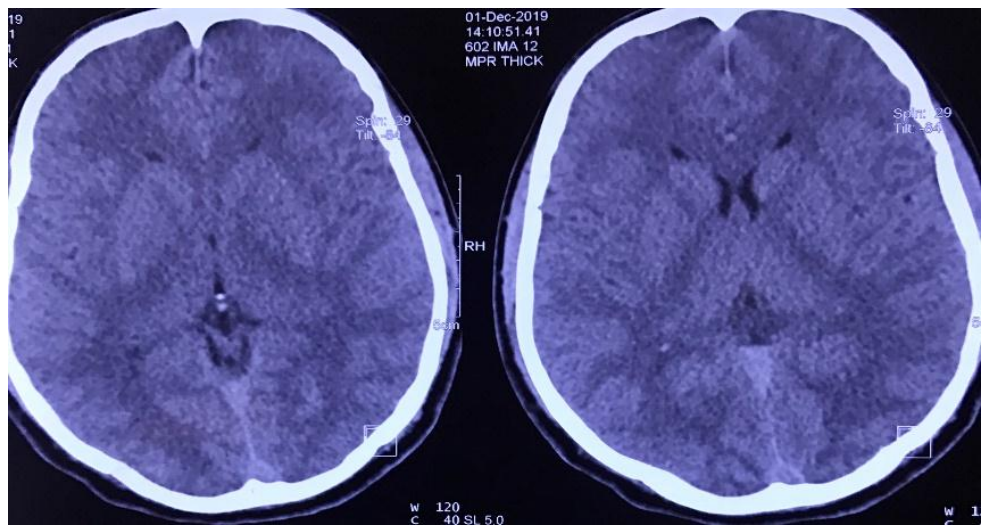

Figure4. CT scan of head

On the day 3 of intubation, he started to desaturate again (SPO2-72\%) then the ventilator parameters were changed and portable chest $\mathrm{x}$ ray showed collapsed right upper and middle lobe. Fibreoptic bronchoscopy (figure 5) was performed which revealed a mucus plug and blood clot in the right upper main bronchus, suctioning was done.

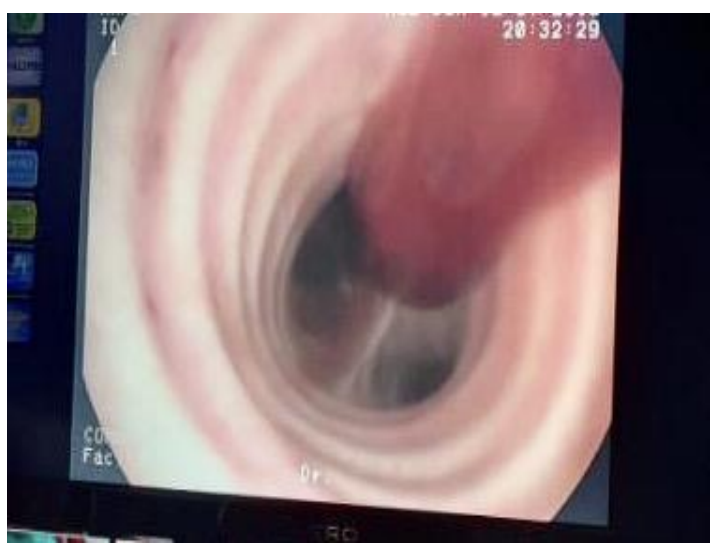

Figure5. bronchoscopy: blood clot in the right upper main bronchus

Patient received 3 pints of packed red cell volume as his hemoglobin dropped from $13.7 \mathrm{gm} \%$ at admission to minimum of $7 \mathrm{gm} \%$. All the other laboratory parameters were within normal range, vitals remained stable, and patient was gradually weaned off the ventilator and extubated after 6 days of ventilator stay. He maintained oxygenation with face mask. Patient underwent the surgery of both upper and lower limb 4 days post-extubation. Post-operative stay was uneventful and was shifted to orthopedic ward.

\section{Discussion}

Fat embolism syndrome is most commonly associated with orthopedic trauma with highest incidence among closed long bone fractures of the lower extremities. The exact incidence of fat embolism and FES are unknown. Approximately one-half of the patients with fat embolism syndrome caused by long bone fractures develop refractory hypoxemia and respiratory insufficiency and might require mechanical ventilation. [4] Although poorly understood, the development of FES is attributed to a series of 
biochemical cascades resulting from the mechanical insult sustained in major trauma. Release of fat emboli leads to occlusion of the microvasculature, triggering an inflammatory response that is clinically manifested by petechial rashes, pulmonary, and neurologic dysfunction. [5]

The classic triad of neurological dysfunction, hypoxemia, and cutaneous manifestation is usually not present in all patient. There are no universal criteria for diagnosis of FES. Diagnosis is made by clinical suspicion and specific findings on imaging methods. However, there Table1. Gurd and Wilson's criteria have been three previously proposed criteria for the diagnosis of the syndrome. Gurd's criteria (table 1) were used most widely, and the diagnosis of FES requires at least two major criteria or one major criterion plus four minor criteria. [6,7] but this criteria lacked lung pathology descriptions and arterial blood gas findings. In Lindeque's criteria (table 2), FES can be diagnosed using ventilator and arterial blood gas parameters [8] Schonfeld has suggested a scoring system to helping diagnosis (table 3) [9] and none of these criteria have been validated or have been universally adopted.

\begin{tabular}{|c|c|}
\hline Major criteria & Minor criteria \\
\hline 1. Petechial rash & 1. Tachycardia (HR 120bpm) \\
\hline 2. Respiratory symptom with radiographic change & 2. Pyrexia (temperature $>39^{\circ} \mathrm{C}$ ) \\
\hline $\begin{array}{l}\text { 3. Central nervous system sign unrelated to trauma } \\
\text { or other conditions }\end{array}$ & $\begin{array}{l}\text { 3. Retinal change (fat or petechiae) } \\
\text { 4. Acute thrombocytopenia } \\
\text { 5. Acute decrease in hemoglobin } \\
\text { 6. High erythrocyte sedimentation rate (ESR) } \\
\text { 7. Fat globules in sputum }\end{array}$ \\
\hline
\end{tabular}

Table2. Lindeque's Criteria

1. Sustained $\mathrm{PaO} 2<8 \mathrm{kPa}$

2. Sustained $\mathrm{PaCO} 2>7.3 \mathrm{kPa}$ or $\mathrm{pH}<7.3$

3. Sustained respiratory rate $>35 / \mathrm{min}$ despite sedation

4. Increase work of breathing, dyspnea, accessory muscle use, tachycardia, and anxiety.

Table3. Schonfeld's criteria (total score $>5$ required for diagnosis)

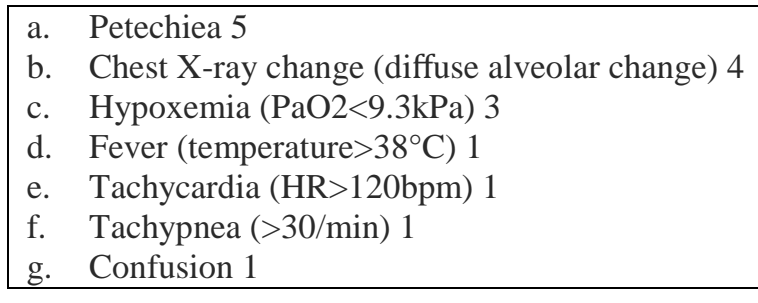

Our case completely fulfilled all the criteria for the diagnosis of fat embolism syndrome. CT scan of brain was normal, however patient had shown some irritability, anxiety and confusion. This altered mentation might due to changing gas analysis. CT scan of the head may be helpful in excluding traumatic causes of altered behavior, its use in FES is limited [2]. MRI of the brain is sensitive for detecting FES.

Supportive care in intensive care unit is standard in FES. Most patients are severely hypovolemic to reversibly tachycardic or irreversibly hypovolemic requiring fluid resuscitation. Supportive correction of hypoxemia with supplemental oxygen or mechanical ventilation is often necessary. Patient might degrade into full blown acute respiratory distress syndrome. Corticosteroid therapy has been proposed as a beneficial therapy for FES by limiting free fatty acid levels, stabilizing membranes, and inhibiting complement mediated leukocyte aggregation. Corticosteroid has reduced the risk of FES by $77 \%$ in a meta-analysis of seven randomized trials that had used prophylactic corticosteroids in patients with long-bone fractures. [10, 11]

In cases of fulminant FES, corticosteroids may be considered, however, there is insufficient evidence to support routine administration of corticosteroids for the majority of patients with FES. Systemic anticoagulation has also been considered as an auxiliary therapy for FES as heparin stimulates lipase activity and therefore may accelerate the clearance of lipids from circulation, but the resultant increase in free fatty acids could exacerbate the underlying proinflammatory physiology. High-dose Rosuvastatin specifically for its antiinflammatory effects has also been claimed to 
treat a patient with severe fat embolism syndrome with neurological manifestation. [12]

\section{CONCLUSION}

There is no specific diagnostic method or universally employed standard criteria for FES. A high degree of suspicion, combined with continuous monitoring of vitals is needed in orthopedic trauma patients with long bone involvement. Treatment is largely supportive which involves judicious fluid resuscitation, oxygenation or mechanical ventilation. Symptoms are often transient with good prognosis and complete recovery is expected with adequate supportive care. Due to the improved critical care, mortality is below $10 \%$.

\section{REFERENCES}

[1] Habashi NM, Andrews PL, Scalea TM. Therapeutic aspects of fat embolism syndrome. Injury. 2006 Oct. 37 Suppl 4:S68-73.

[2] Mellor A, Soni N. Fat embolism. Anaesthesia. 2001;56:145-154

[3] Stein PD, Yaekoub AY, Matta F, Kleerekoper M. Fat embolism syndrome. Am J Med Sci 2008; 336:472.

[4] King MB Harmon KR Unusual forms of pulmonary embolism. Clin Chest Med 1994 Sep; 15(3):561-80.

[5] Meyer N, Pennington WT, Dewitt D, Schmeling GJ. Isolated cerebral fat emboli syndrome in multiply injured patients: a review of three cases and the literature. J Trauma. 2007 Dec. 63(6):1395-402.
[6] Gurd AR and Wilson RI, "The fat embolism syndrome," Journal of Bone and Joint Surgery, 1971; 56(3): 408-416.

[7] Michael E. Kwiatt, Mark J. Seamon. Fat embolism syndrome. International Journal of Critical Illness and Injury Science .2013 janmar; 3 (1):64-68.

[8] Lindeque BG, Schoeman HS, Dommisse GF, Boeyens MC, and Vlok AL, Fat embolism and the fat embolism syndrome. A double-blind therapeutic study, Journal of Bone and Joint Surgery, 1987; 69(1): 128-131.

[9] Schonfeld SA, Ploysongsang Y, DiLisio R etal. Fat embolism prophylaxis with corticosteroids. A prospective study in high-risk patients. Annals of Internal Medicine, 1983; 99(4): 438443.

[10] Bederman SS, Bhandari M, McKee MD, Schemitsch EH. Do corticosteroids reduce the risk of fat embolism in patient with long bone fractures? A meta-analysis. Can J Surg 2009; 52:386-93.

[11] Douglas FS, César VC, Thiago RAC, Gustavo P F, Bartolomeu N, SandroR. The use of corticosteroid for the prophylaxis of fat embolism syndrome in patients with long bone fracture. Rev. Col. Bras. Cir. 2013; 40(5): 423426.

[12] Whalen LD, Khot SP, Standage SW. High-dose rosuvastatin treatment for multifocal stroke in trauma-induced cerebral fat embolism syndrome: a case report. Pediatr Neurol. 2014 Sep; 51(3):410-3.

Citation: Moktan, Sushila Lama. Post Traumatic Fat Embolism Syndrome: A Case Report ARC Journal of Anesthesiology. 2019; 4(2): 19-23. doi:dx.doi.org/ 10.20431/2455-9792.0402004.

Copyright: () 2019 Authors. This is an open-access article distributed under the terms of the Creative Commons Attribution License, which permits unrestricted use, distribution, and reproduction in any medium, provided the original author and source are credited. 